\title{
Laser-stimulated deexcitation of Rydberg antihydrogen atoms
}

\author{
D. Comparat \\ Laboratoire Aimé Cotton, CNRS, Université Paris-Sud, ENS Paris Saclay, Université Paris-Saclay, Bâtiment 505, 91405 Orsay, France \\ C. Malbrunot \\ Experimental Physics Department, CERN, Genève 23, 1211, Switzerland
}

(Received 16 October 2018; published 16 January 2019)

\begin{abstract}
Antihydrogen atoms are routinely formed at CERN in a broad range of Rydberg states. Ground-state antiatoms, those useful for precision measurements, are eventually produced through spontaneous decay. However given the long lifetime of Rydberg states the number of ground-state antihydrogen atoms usable is small, in particular for experiments relying on the production of a beam of antihydrogen atoms. Therefore, it is of high interest to efficiently stimulate the decay in order to retain a higher fraction of ground-state atoms for measurements. We propose a method that optimally mixes the high angular momentum states with low ones enabling us to stimulate, using a broadband frequency laser, the deexcitation toward low-lying states, which then spontaneously decay to the ground state. We evaluate the method in realistic antihydrogen experimental conditions. For instance, starting with an initial distribution of atoms within the $n=20-30$ manifolds, as formed through the charge exchange mechanism, we show that more than $80 \%$ of antihydrogen atoms will be deexcited to the ground state within $100 \mathrm{~ns}$ using a laser producing $2 \mathrm{~J}$ at $828 \mathrm{~nm}$.
\end{abstract}

DOI: 10.1103/PhysRevA.99.013418

\section{INTRODUCTION}

Recent breakthroughs were achieved in spectroscopy measurements on antihydrogen atoms which led to stringent tests of the CPT symmetry, the combination of the charge conjugation, parity, and time reversal symmetries [1-3]. These measurements were all performed on magnetically trapped ground-state antihydrogen atoms which is currently the only method succeeding in accumulating enough ground-state atoms for measurements. Indeed, antihydrogen atoms formed at CERN, using three-body recombination or charge exchange processes, are produced in a broad range of Rydberg states including all possible angular momentum states $[4,5]$. The highly excited atoms must first decay before a precision measurement can be performed. Trapped atoms can be held on for hours [6] so that the produced Rydberg atoms have ample time to decay. In beam experiments however, the spontaneous lifetimes of the Rydberg states are hindering a fast enough ground-state population [7]. Neglecting first the effect of external fields on the spontaneous lifetime of a $(n, l, m)$ state (where $|m| \leqslant l<n$ ), the lifetime can be approximated by [8]

$$
\tau \approx\left(\frac{n}{30}\right)^{3}\left(\frac{l+1 / 2}{30}\right)^{2} \times 2.4 \mathrm{~ms}
$$

Published by the American Physical Society under the terms of the Creative Commons Attribution 4.0 International license. Further distribution of this work must maintain attribution to the author(s) and the published article's title, journal citation, and DOI.
This result is also confirmed in a magnetic field environment. For instance Ref. [9] shows that within the 1-5 T field present in antihydrogen experiments and for the threebody recombination formation mechanism, only $10 \%$ of the population with $n<30$ reach the ground state in $100 \mu \mathrm{s}$. A much longer time $(\sim 2 \mathrm{~ms})$ is required to have $50 \%$ of the population reaching the ground state due to the large proportion of states with high angular momentum. Because antihydrogen atoms are typically formed with velocities of $1000 \mathrm{~ms}^{-1}$ (corresponding to the mean velocity of a MaxwellBoltzmann distribution at $\sim 50 \mathrm{~K}$ ), if they are not trapped, they will hit the walls of the formation apparatus and annihilate long before any spontaneous deexcitation to the ground state can occur.

It is therefore of high interest to enhance the decay. It has been suggested that coupling or mixing high angular momentum states with low ones may accelerate the decay [10]. The main idea of the present article is to mix all angular momenta using an electric field added to the already present 1-5 $\mathrm{T}$ magnetic field of the antihydrogen experiments and use a laser to stimulate the decay from high-lying $n$ states to a deep-lying one with a short spontaneous lifetime. The principle of the proposed scheme is sketched in Fig. 1.

We first estimate the feasibility of the method using a simple model assuming a fully mixed system and confirm that the laser power required is compatible with existing lasers and that photoionization can be drastically reduced by choosing a low enough $n^{\prime}$ manifold. We then discuss the validity of the first-order treatment in the electric and magnetic fields and show that an optimal configuration of fields can lead to a large mixing of the states. Finally using this optimal configuration of fields we study in more detail the effect of the laser power and polarization. 


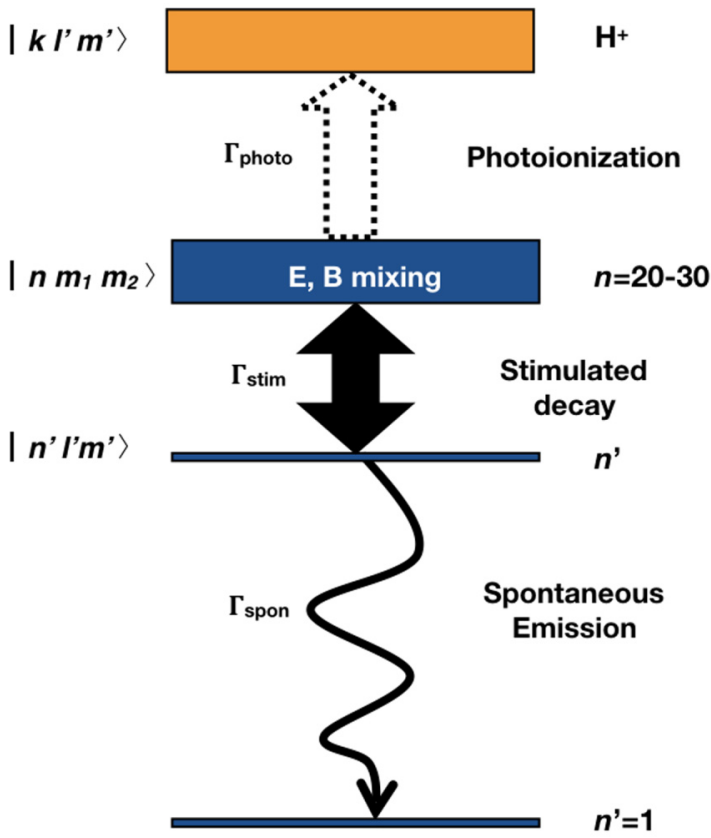

FIG. 1. Principle of the stimulated decay of Rydberg antihydrogen atoms. Combined electric $\boldsymbol{E}$ and magnetic $\boldsymbol{B}$ fields mix the Rydberg states to form new $\left|n m_{1} m_{2}\right\rangle$ states that can then be stimulated down to $n^{\prime}$ at a rate $\Gamma_{\text {stim. }}$. This state will quickly decay toward the ground state. The other basis states and the competing photoionization mechanism are shown.

\section{GENERAL CONSIDERATIONS ASSUMING FULLY MIXED STATES}

For the sake of simplicity we focus here on antihydrogen formed in a pulsed charge exchange process [11]. The other formation mechanism used by the antihydrogen experiments is based on "continuous" three-body recombination of positrons and antiprotons (lasting as long as the antiproton and positron clouds can be kept in interaction, typically several hundreds of milliseconds) producing typically $n>40[4,5]$. This "continuous" production mode will be the focus of another publication where we will show that $\mathrm{THz}$ light can be efficiently used to stimulate the decay [12]. Consequently, we assume an initial distribution of $n=20-30$ states with full degeneracy in $l$ and $m$ as planned to be produced in the AEgIS experiment [11].

Before studying in detail the mixing mechanism in Sec. III we assume the presence of an electric and magnetic field resulting in a perfect state mixing. We can already see that such hypothesis is likely to be valid by estimating the firstorder Zeeman and Stark effects (see Sec. III A) which indicate that for $n \sim 20-30$ (with $r \sim n^{2} a_{0}$ ) an electric field of the order of $F \sim 100 \mathrm{~V} / \mathrm{cm}$ is sufficient to produce a Stark effect bigger than the spacing between Zeeman sublevels leading to a strong mixing of the (Zeeman) $|n l m\rangle$ states.

The assumption of perfect state mixing will allow us to evaluate the laser properties needed to stimulate the decay. The first obvious requirement is that the laser has to be broadband: in order to cover all Rydberg states $n=20-30$ we need a laser linewidth on the order of $\sim 2 \pi \times 5000 \mathrm{GHz}$. Note that with such bandwidth we can also cover $n=25-\infty$.

\section{A. Fully mixed states hypothesis}

We assume a fully $l, m$ mixed initial state $\left|\psi_{n}\right\rangle \approx$ $\sum_{l m} 1 / n|n l m\rangle$ that is coupled to the lower $n^{\prime}$ manifold thanks to a (spectrally Lorentzian) laser of FWHM $\Gamma_{\mathrm{L}}=2 \pi \times$ $5000 \mathrm{GHz}$ and of central wavelength $\lambda=2 \pi c / \omega$. We first consider an isotropic polarization of the light. We can then calculate the stimulated emission and photoionization rates for a given laser intensity $I$.

\section{Stimulated emission and photoionization rates}

The stimulated emission rate $\Gamma_{\text {stim }}$ under an unpolarized light is given by the sum over all polarizations $q$ and over all final states $l^{\prime} m^{\prime}: \Gamma_{\text {stim }}=$ $\frac{2 I e^{2} a_{0}^{2}}{\hbar^{2} \epsilon_{0} c \Gamma_{\mathrm{L}}} \frac{1}{3} \sum_{q} \sum_{l^{\prime} m^{\prime}}\left|\left\langle\psi_{n}\left|r^{(q)} / a_{0}\right| n^{\prime} l^{\prime} m^{\prime}\right\rangle\right|^{2}=\frac{2 I e^{2} a_{0}^{2}}{\hbar^{2} \epsilon_{0} c \Gamma_{\mathrm{L}}} \frac{1}{3 n^{2}} \sum_{l^{\prime} l}$ $\left|\left\langle n^{\prime} l^{\prime}\left\|r / a_{0}\right\| n l\right\rangle\right|^{2}$, with $\left\langle n^{\prime} l^{\prime}\left\|r / a_{0}\right\| n l\right\rangle=C_{l 0,10}^{l^{\prime} 0} \sqrt{2 l+1} R_{n l}^{n^{\prime} l^{\prime}}$, where $R_{n l}^{n^{\prime} l^{\prime}}$ is the radial overlap given in Eq. (A4) of the Appendix.

Similarly, using the extra photon energy above the ionization threshold given by $E=k^{2} R y=\hbar \omega-R y / n^{2}$, we find the photoionization cross section: $\sigma_{n}^{k}=$ $\frac{1}{3} \sum_{q} \sum_{l^{\prime} m^{\prime}} 4 \pi^{2} \alpha a_{0}^{2}\left(\frac{1}{n^{2}}+k^{2}\right)\left|\left\langle k l^{\prime} m^{\prime}\left|r^{(q)} / a_{0}\right| \psi_{n}\right\rangle\right|^{2}=$

$\frac{1}{n^{2}} \sum_{l l^{\prime}}(2 l+1) \sigma_{n l}^{k l^{\prime}}$, where $\sigma_{n l}^{k l^{\prime}}$ is the photoionization cross section from $n l$ to $k l^{\prime}$ given by $\sigma_{n l}^{k l^{\prime}}=$ $\frac{4 \pi^{2} \alpha a_{0}^{2}}{3}\left(\frac{1}{n^{2}}+k^{2}\right) \frac{\max \left(l, l^{\prime}\right)}{2 l+1}\left(R_{n l}^{k l^{\prime}}\right)^{2} . \quad R_{n l}^{k l^{\prime}}$ is the radial overlap given by Eqs. (A8) and (A9) in the Appendix.

Finally, the photoionization rate is $\Gamma_{\text {photo }}=\int d \tilde{\omega} \frac{I(\tilde{\omega})}{\hbar \tilde{\omega}} \sigma_{n}^{k(\tilde{\omega})}$ and, because the cross section (and also the $\omega$ value) does not vary significantly over the laser spectral bandwidth we have $\Gamma_{\text {photo }}=\frac{I}{\hbar \omega} \sigma_{n}^{k}$.

\section{Saturation energy required}

In order to have an efficient transfer toward the ground state we need to transfer the population in a time scale compatible with the laser pulse and the spontaneous emission lifetime of the $n^{\prime}$ levels.

We study two extreme cases. The first one assumes a short nanosecond (10 ns) laser pulse for which we calculate the saturated intensity $I=I_{\text {sat }}(10 \mathrm{~ns})$ such that the stimulated decay rate is $\Gamma_{\text {stim }}=1 /(10 \mathrm{~ns})$. In the second case we simply require a pulse duration comparable to the spontaneous emission lifetime $\tau$ of the $n^{\prime}$ manifold and calculate the saturated intensity $I_{\text {sat }}(\tau)$ such that the stimulated decay rate is $1 / \tau$. Obviously $I_{\text {sat }}(10 \mathrm{~ns})=I_{\text {sat }}(\tau) \frac{\tau}{10 \mathrm{~ns}}$, but we find it useful to indicate both values. For this first study we choose $\tau^{-1}=\frac{1}{n^{\prime 2}} \sum_{l^{\prime}=0}^{n^{\prime}-1}\left(2 l^{\prime}+1\right) A_{n^{\prime} l^{\prime}}$ which is the average decay rate (over all $l^{\prime} m^{\prime}$ levels) of the $n^{\prime}$ manifold. It is calculated using $A_{n l}=\sum_{n^{\prime}=1}^{n-1}\left(A_{n l}^{n^{\prime} l+1}+A_{n l}^{n^{\prime} l-1}\right)$ where $A_{n l}^{n^{\prime} l^{\prime}}=$ $\frac{\alpha^{4} c}{6 a_{0}}\left(\frac{1}{n^{\prime 2}}-\frac{1}{n^{2}}\right)^{3} \frac{\max \left(l, l^{\prime}\right)}{2 l+1}\left(R_{n l}^{n^{\prime} l^{\prime}}\right)^{2}$ is the spontaneous emission rate from an $n l$ state toward all $n^{\prime} l^{\prime}$ sub-levels ( $\hbar \omega=\frac{R y}{n^{\prime 2}}-\frac{R y}{n^{2}}$ is the transition energy).

The results on the obtained required saturation energy, assuming a laser waist of $1 \mathrm{~mm}$ (similar to the typical antiproton plasma size; see, e.g., [13]), as well as the ratio $\frac{\Gamma_{\text {stim }}}{\Gamma_{\text {photo }}}$, are shown in Table I and Fig. 2 .

We first see that the competing effect of the photoionization toward the continuum is relatively weak especially for low 
TABLE I. Study of stimulated deexcitation. The laser linewidth $\Gamma_{\mathrm{L}}$ is assumed to be $2 \pi \times 5000 \mathrm{GHz}$. The laser waist is $1 \mathrm{~mm}$.

\begin{tabular}{ccccccc}
\hline \hline$n$ & $n^{\prime}$ & $\lambda(\mathrm{nm})$ & $\tau(\mathrm{ns})$ & $E_{\mathrm{sat}}(\tau)(\mathrm{mJ})$ & $E_{\text {sat }}(10 \mathrm{~ns})(\mathrm{mJ})$ & $\frac{\Gamma_{\text {stim }}}{\Gamma_{\text {photo }}}$ \\
\hline 30 & 10 & 10250 & 1908 & 0.0043 & 0.82 & 0.78 \\
20 & 10 & 12150 & 1908 & 0.00029 & 0.056 & 0.78 \\
30 & 5 & 2343 & 86.5 & 4.4 & 38.5 & 6.0 \\
20 & 5 & 2430 & 86.5 & 0.51 & 4.4 & 6.0 \\
30 & 4 & 1484 & 33 & 37 & 124 & 11.4 \\
20 & 4 & 1518.8 & 33 & 4.5 & 14.9 & 11.4 \\
30 & 3 & 828.4 & 10 & 548 & 548 & 26.3 \\
20 & 3 & 839.0 & 10 & 68.7 & 68.7 & 26.3 \\
30 & 2 & 366.1 & 2.1 & 20708 & 4405 & 84.6 \\
20 & 2 & 368.2 & 2.1 & 2670 & 568 & 84.6 \\
\hline \hline
\end{tabular}

$n^{\prime}$ values. This is due to the fact that the photoionization cross section drops quickly for high angular momentum states which are the most numerous states and that lower $n^{\prime}$ implies shorter laser wavelength that also reduces the photoionization effect.

Obviously, the power required to deexcite increases with decreasing $n^{\prime}$ due to the $n^{13 / 2}$ scaling of the dipole matrix element and to the fact that fewer angular momenta $\left(\left|m^{\prime}\right| \leqslant\right.$ $l^{\prime}<n^{\prime}$ ) exist and can be coupled to the initial ones.

Based on these results we see that several laser choices are possible. Toward $n^{\prime}=10$, power in the watt range is enough and some $\mathrm{CO}_{2}$ lasers exist that can even allow a continuous deexcitation scheme for high $n$ values [14,15]. But lower $n^{\prime}$ are better to minimize photoionization. Deexcitation toward $n^{\prime}=4$ seems feasible using a laser similar to the one used by AEgIS to excite positronium (a bound state of electron and positron) to Rydberg states [16]. However the use of a nanosecond laser with a pulse much shorter than the spontaneous emission lifetime of the $n^{\prime}$ states will limit the transfer (a)

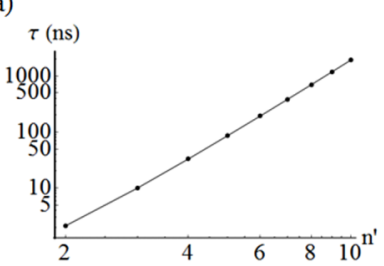

(c)

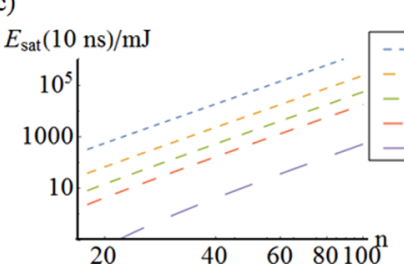

(b)

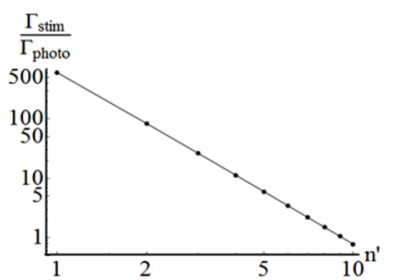

(d)

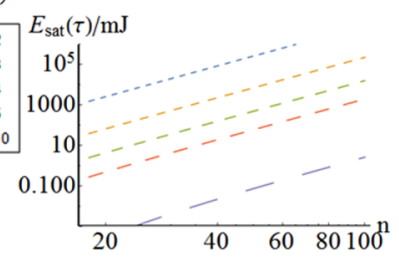

FIG. 2. (a) Average lifetime $\tau$ of the $n^{\prime}$ manifold. (b) Ratio of stimulated emission to photoionization rates. (c) Energy required to saturate the (fully mixed) $n \rightarrow n^{\prime}$ transition: $E_{\text {sat }}(10 \mathrm{~ns})$ for an interaction time of $10 \mathrm{~ns}$. (d) $E_{\mathrm{sat}}(\tau)$ for an interaction time of $\tau$. As above the laser linewidth $\Gamma_{\mathrm{L}}$ is assumed to be $2 \pi \times 5000 \mathrm{GHz}$ and the waist $1 \mathrm{~mm}$. We find the approximate analytic formulas: $\tau(\mathrm{ns}) \approx 0.065 n^{\prime 4.5}, E_{\mathrm{sat}}(\tau)(\mathrm{mJ}) \approx 0.77 n^{5} n^{\prime-9.5}, E_{\text {sat }}(10 \mathrm{~ns})(\mathrm{mJ})$ $\approx 0.007 n^{\prime-5.2} n^{5}, \frac{\Gamma_{\text {stim }}}{\Gamma_{\text {photo }}} \approx 630 n^{\prime-2.9}$. by equalizing population between upper and lower levels. Therefore, targeting a lower state, like $n^{\prime}=3$, will improve the deexcitation efficiency. Furthermore intense lasers that have a long pulse duration $(\sim 100 \mathrm{~ns})$ in the joule range exist at this wavelength. This is the case for alexandrite [17] which can reach $\lambda>800 \mathrm{~nm}$ through heating of the medium [18].

\section{MIXING IN ELECTRIC AND MAGNETIC FIELDS}

For the next study we therefore restrict ourselves to $n^{\prime}=3$, but most of the results will be valid for any other case.

Here we study in more detail the mixing produced by an electric and a magnetic field. We neglect the spins because Stark and Zeeman effects in the Rydberg $n$ manifold usually dominate the fine or hyperfine effects.

\section{A. First-order Stark and Zeeman effects}

Considering a given $n$ manifold, we have the following Hamiltonian:

$$
H=-\frac{1}{2 n^{2}}+\boldsymbol{r} \cdot \boldsymbol{F}+\frac{1}{2} \boldsymbol{B} \cdot \boldsymbol{L}-\frac{1}{8}(\boldsymbol{r} \times \boldsymbol{B})^{2} .
$$

Atomic units will be assumed in this section.

Here, we use perturbation theory in the field values (not to be confused with the standard state perturbation theory). To the first order in the fields' values we simply have to deal with the perturbation $V_{1}=\boldsymbol{r} \cdot \boldsymbol{F}+\frac{1}{2} \boldsymbol{B} \cdot \boldsymbol{L}$. We can indeed neglect the second-order term since it is on the order of $n^{6} F^{2}+n^{4} B^{2} / 4$ in atomic units [so for $B /\left(2.35 \times 10^{5} \mathrm{~T}\right)$ and $\left.F /\left(5.14 \times 10^{9} \mathrm{~V} / \mathrm{cm}\right)\right]$ [19-21]. Thus, the second-order becomes comparable to the first order [that is on the order of $(B+3 n F) n / 2$ ] for $n \leqslant 30$ and $F<1 \mathrm{kV} / \mathrm{cm}$ for a $1-5 \mathrm{~T}$ field. Therefore, in our configuration, the first order should be accurate enough to extract the required fields values and laser energy.

The Hamiltonian (1) has been studied by Pauli who showed that, for a given $n$ manifold, $\boldsymbol{r}=-\frac{3}{2} n \boldsymbol{A}$, where $\boldsymbol{A}$ is the Runge-Lenz vector. So in this manifold we can define new angular momenta $\boldsymbol{I}_{1}=\frac{\boldsymbol{L}+\boldsymbol{A}}{2}$ and $\boldsymbol{I}_{2}=\frac{\boldsymbol{L}-\boldsymbol{A}}{2}$ that commute and verify $I_{1}=I_{2}=\frac{n-1}{2}$. We will use the $\left|I_{1} m_{1}\right\rangle \otimes\left|I_{2} m_{2}\right\rangle$ basis where the eigenvalues $m_{1}, m_{2}$ (on a given axis) take the values $-(n-1) / 2,-(n-3) / 2, \ldots,(n-1) / 2$. We define $\omega_{1}=$ $\frac{B-3 n \boldsymbol{F}}{2}$ and $\boldsymbol{\omega}_{2}=\frac{B+3 n \boldsymbol{F}}{2}$ such that $V_{1}=\boldsymbol{\omega}_{1} \cdot \boldsymbol{I}_{1}+\boldsymbol{\omega}_{2} \cdot \boldsymbol{I}_{2}$. That is trivial to diagonalize using the $\left|I_{1} m_{1}\right\rangle_{\omega_{1}}\left|I_{2} m_{2}\right\rangle_{\omega_{2}}=$ $\left|n m_{1} m_{2}\right\rangle$ basis. This notation indicates that $m_{1}$ is the projection of $I_{1}$ on the $\omega_{1}$ axis. So the first-order perturbation theory gives

$$
\Delta E_{m_{1} m_{2}}^{(1)}=\omega_{1} m_{1}+\omega_{2} m_{2},
$$

where $\omega_{i}=\left\|\boldsymbol{\omega}_{i}\right\|$.

For a pure electric field we restore the pure Stark effect $\Delta E_{m_{1} m 2}^{(1)}=\frac{3}{2} n F\left(m_{1}+m_{2}\right)$ with a clear relation to the parabolic basis $|n, k, m\rangle$ linked to $\hat{H}, \hat{A}_{z}, \hat{L}_{z}$ eigenvalues: $k=$ $-\left(m_{1}+m_{2}\right)$ and $m=m_{2}-m_{1}[19,22]$.

In a pure magnetic field we restore the standard Zeeman shift $\Delta E_{m_{1} m_{2}}^{(1)}=\frac{1}{2} B\left(m_{1}+m_{2}\right)$, where $m_{1}+m_{2}=m$ because $\boldsymbol{I}_{1}+\boldsymbol{I}_{2}=\boldsymbol{L}$.

Using $\boldsymbol{L}=\boldsymbol{I}_{1}+\boldsymbol{I}_{2}$ and the standard sum of the two angular momenta leads to $|n l m\rangle_{\boldsymbol{B}}=$ 
$\sum_{m_{1} m_{2}} C_{I_{1} m_{1}, I_{2} m_{2}}^{l m}\left|I_{1} m_{1}\right\rangle_{\boldsymbol{B}}\left|I_{2} m_{2}\right\rangle_{\boldsymbol{B}} \quad$ or $\quad\left|I_{1} m_{1}\right\rangle_{\boldsymbol{B}}\left|I_{2} m_{2}\right\rangle_{\boldsymbol{B}}=$ $\sum_{m l} C_{I_{1} m_{1}, I_{2} m_{2}}^{l m}|n l m\rangle_{\boldsymbol{B}}=\sum_{l} C_{I_{1} m_{1}, I_{2} m_{2}}^{l, m_{1}+m_{2}}\left|n l, m=m_{1}+m_{2}\right\rangle_{\boldsymbol{B}}$, where the subscript ${ }_{\boldsymbol{B}}$ indicates that the quantization axis $z$ is along $\boldsymbol{B}$.

If we define $\alpha_{1}$ and $\alpha_{2}$ as the angles between the magnetic field $\boldsymbol{B}$ axis and the vectors $\boldsymbol{\omega}_{1}$ and $\boldsymbol{\omega}_{2}$, respectively, by the use of the Wigner $D$ rotation matrix, we have [22] (Eq. 1.4 (35) of [23])

$$
\left|I_{1} m_{1}\right\rangle_{\omega_{1}}=\sum_{m_{1}^{\prime}=-I_{1}}^{m_{1}^{\prime}=I_{1}} D_{m_{1}, m_{1}^{\prime}}^{I_{1}}\left(0, \alpha_{1}, 0\right)\left|I_{1} m_{1}^{\prime}\right\rangle_{\boldsymbol{B}} .
$$

So by combining the equations [we use real CleschGordan and real Wigner (small) $d$ matrix $d_{m_{2}, m_{2}^{\prime}}^{I_{2}}\left(\alpha_{2}\right)=$ $\left.D_{m_{2}, m_{2}^{\prime}}^{I_{2}}\left(0, \alpha_{2}, 0\right)\right]$ we get

$$
\begin{aligned}
\left|n m_{1} m_{2}\right\rangle_{\omega_{1}, \omega_{2}} & \equiv\left|I_{1} m_{1}\right\rangle_{\omega_{1}}\left|I_{2} m_{2}\right\rangle_{\omega_{2}} \\
& =\sum_{l=0}^{n-1} \sum_{m=-l}^{l}\left\langle n l m \mid n m_{1} m_{2}\right\rangle|n l m\rangle_{\boldsymbol{B}} \\
\left\langle n l m \mid n m_{1} m_{2}\right\rangle \equiv & \sum_{m_{1}^{\prime}=-I_{1}}^{m_{1}^{\prime}=I_{1}=\frac{n-1}{2}} \sum_{m_{2}^{\prime}=-I_{2}}^{m_{2}^{\prime}=I_{2}=\frac{n-1}{2}} d_{m_{1}, m_{1}^{\prime}}^{I_{1}}\left(\alpha_{1}^{(n)}\right) d_{m_{2}, m_{2}^{\prime}}^{I_{2}}\left(\alpha_{2}^{(n)}\right) \\
& \times C_{I_{1} m_{1}^{\prime}, I_{2} m_{2}^{\prime}}^{l m},
\end{aligned}
$$

where we have written $\alpha_{1}^{(n)}$ to stress that the angles depend on $n$ and not only on the fields' values. Similarly $|n l m\rangle_{\boldsymbol{B}}=\sum_{m_{1}, m_{2}}\left\langle n m_{1} m_{2} \mid n l m\right\rangle\left|n m_{1} m_{2}\right\rangle_{\boldsymbol{\omega}_{1}, \boldsymbol{\omega}_{2}}$. And we have the closure expression $\mathbf{1}_{n}=\sum_{m_{1}, m_{2}}\left|n m_{1} m_{2}\right\rangle\left\langle n m_{1} m_{2}\right|=$ $\sum_{l m}|n l m\rangle_{\boldsymbol{B} \boldsymbol{B}}\langle n l m|$.

We can note that using such an $\left(m_{1}, m_{2}\right)$ formalism the states will always be given in a parabolic basis, not in a spherical basis, so even in a pure magnetic field the eigenstates $\left|n m_{1} m_{2}\right\rangle$ will not correspond to the $|n l m\rangle$ ones: $l$ is mixed in the $\left|n m_{1} m_{2}\right\rangle$ basis.

\section{B. Laser transitions under combined electric and magnetic fields}

In order to choose the electric field which most optimally mixes the states, we need to calculate all transitions dipole moments from a given $\left|n m_{1} m_{2}\right\rangle$ toward each states of the $n^{\prime}$ manifold. The Zeeman and Stark effect being small for $n^{\prime}=3$ we use the $\left|n^{\prime} l^{\prime} m^{\prime}\right\rangle$ basis. So the stimulated emission rate, for a $q= \pm 1,0$ polarization, from an $\left|n m_{1} m_{2}\right\rangle$ state toward all the $n^{\prime}$ manifolds is given by

$$
\begin{aligned}
& \Gamma_{\text {stim }}^{n m_{1} m_{2} ; n^{\prime} ; q}=\frac{2 I e^{2} a_{0}^{2}}{\hbar^{2} \epsilon_{0} c \Gamma_{\mathrm{L}}} \sum_{l^{\prime}, m^{\prime}}\left|\left\langle n^{\prime} l^{\prime} m^{\prime}\left|\frac{r^{(q)}}{a_{0}}\right| n m_{1} m_{2}\right\rangle\right|^{2} \\
& =\frac{2 I e^{2} a_{0}^{2}}{\hbar^{2} \epsilon_{0} c \Gamma_{\mathrm{L}}} \sum_{m, l^{\prime}}\left|\sum_{l=l^{\prime} \pm 1}\left\langle n^{\prime} l^{\prime} m+q\left|\frac{r^{(q)}}{a_{0}}\right| n l m\right\rangle\left\langle n l m \mid n m_{1} m_{2}\right\rangle\right|^{2}
\end{aligned}
$$

which can be calculated using Eq. (A3) of the Appendix and Eq. (4) in Sec. III A.

The laser-driven evolution of these states may be quite complex with $n^{2}$ levels coupled to $n^{\prime 2}$ ones (and to the continuum). Rate equations are sufficient to treat the problem assuming that the broadband laser used to stimulate the deexcitation is incoherent (which is likely to be the case). Furthermore, because we have chosen the $n^{\prime}=3$ with a fast spontaneous emission lifetime, the population of the $n^{\prime}=3$ levels will be small and the reexcitation process from $n^{\prime}=3$ to the $n$ manifold will not be severe. We can therefore consider that the $n m_{1} m_{2}$ levels are isolated from each other and thus simplify the picture to a four-level rate equation system as shown in Fig. 1:

$$
\begin{aligned}
\frac{d N_{\mathrm{H}^{+}}}{d t} & =\Gamma_{\text {photo }} N_{n}, \\
\frac{d N_{n}}{d t} & =-\left(\Gamma_{\text {stim }}+\Gamma_{\text {photo }}\right) N_{n}+\Gamma_{\text {stim }} N_{n^{\prime}}, \\
\frac{d N_{n^{\prime}}}{d t} & =\Gamma_{\text {stim }}\left(N_{n}-N_{n^{\prime}}\right)-\Gamma_{\text {spon }} N_{n^{\prime}}, \\
\frac{d N_{1}}{d t} & =\Gamma_{\text {spon }} N_{n^{\prime}} .
\end{aligned}
$$

A cumbersome analytical solution exists for $P_{n m_{1} m_{2}}^{(q)}(t)$, the transfer of a given $\left|n m_{1} m_{2}\right\rangle$ state to the ground state, and we use it throughout this article. However in this section we can simplify the solution because, as seen in Table I and Fig. 2, we can safely neglect the photoionization. We can also assume an instantaneous spontaneous emission from $n^{\prime}=3$ to the ground state if the laser pulse duration is much longer than the spontaneous emission lifetime of $10 \mathrm{~ns}$ (which can be considered the case for an, e.g., 100-ns-long laser pulse as is the case for the alexandrite, for example). In this case, the model becomes a simple two-level model, and the transfer of a given $\left|n m_{1} m_{2}\right\rangle$ state to ground state, after the application of the laser of polarization $q$ and of duration $t$, is given by $P_{n m_{1} m_{2}}^{(q)}(t)=1-e^{-t \Gamma_{\text {stim }}^{n m_{1} m_{2} ; n^{\prime} ; q}}$.

Assuming an equidistribution of the initial $\left|n m_{1} m_{2}\right\rangle$ states we sum over these $n^{2}$ states to get the total number of atoms reaching the ground state. The results are given in Fig. 3 as a function of $\alpha_{1}$ and $\alpha_{2}$ for different laser polarizations. We have used, in Cartesian coordinates with $\boldsymbol{B}=(0,0, B)$, the result $\frac{3 n \boldsymbol{F}}{B}=\left(0,2 \frac{\sin \left(\alpha_{1}\right) \sin \left(\alpha_{2}\right)}{\sin \left(\alpha_{1}+\alpha_{2}\right)}, 2 \frac{\sin \left(\alpha_{1}-\alpha_{2}\right)}{\sin \left(\alpha_{1}+\alpha_{2}\right)}\right)$.

The calculation has been performed for $n=20$ with a laser energy of $\sim 150 \mathrm{~mJ}$ and a pulse of $100 \mathrm{~ns}$ (but similar results hold for other $n$ states or other laser power values).

The first important result is that it is possible to efficiently mix the states by adding an electric field to a magnetic field validating the assumption taken in the previous section. The transfer efficiency is very high for several values and orientations of the electric field. As expected, an unfavorable configuration is that where the fields are orthogonal to each other or in the case of a too small electric field. A typical favorable configuration is when the electric field axis is oriented with a small angle with respect to the magnetic field axis and has a value such that $3 n F \sim B$ in atomic units. So for instance, for $n=30$, in a $1 \mathrm{~T}$ magnetic field, a $280 \mathrm{~V} / \mathrm{cm}$ electric field with $160^{\circ}$ angle (corresponding to $\alpha_{1}=0.187, \alpha_{2}=1.777$ ) is a good choice to efficiently mix the states as shown by the dashed lines in Fig. 3(c). These magnetic and electric field values are small enough to allow the use of the first-order 
(a)

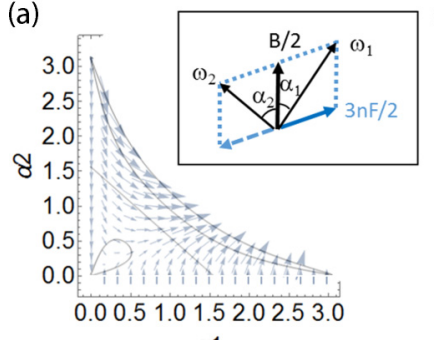

(c)

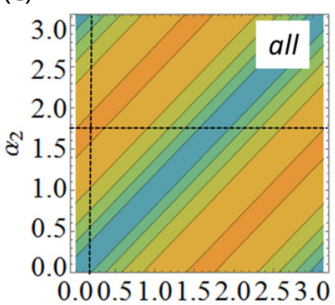

$\alpha_{1}$ (b)

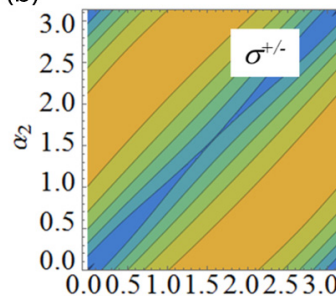

(d)

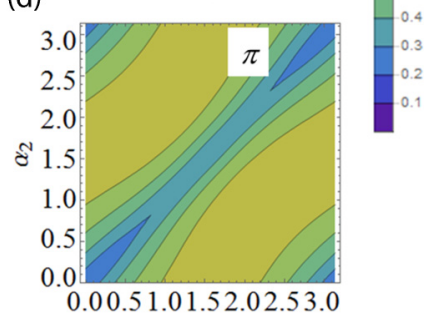

$\alpha_{1}$

FIG. 3. (a) In the inset: Definition of the $\alpha_{1}$ and $\alpha_{2}$ angles. Schematic of the electric $\boldsymbol{F}$ field orientation with respect to the magnetic $\boldsymbol{B}$ field. The frontiers where the length of the $3 n \boldsymbol{F} / B$ vector (in atomic units) is $0.5,1,1.5$, and 2 are shown and no values larger than 2 are plotted. (b), (c), and (d): Fraction $(f)$ of the initial atoms reaching ground state as a function of the mixing induced by an electric field $\boldsymbol{F}$ for the $n=20 \rightarrow n^{\prime}=3$ case and for a laser of $\sim 150 \mathrm{~mJ}$ energy with a pulse duration of $100 \mathrm{~ns}$, a linewidth $\Gamma_{\mathrm{L}}=$ $2 \pi \times 5000 \mathrm{GHz}$, and a waist of $1 \mathrm{~mm}$. For a circular polarized laser (b), for an isotropic laser polarization (c), and for a $\pi$-polarized laser (linear and parallel polarization compared to the $\boldsymbol{B}$ axis) (d). The dashed lines in (c) indicate a judicious choice of $\alpha_{1}$ and $\alpha_{2}$ to achieve maximal mixing and thus maximal ground-state population.

perturbation theory approach. This is indicated by the contour of the $3 n F / B$ values in Fig. 3(a) that should be smaller than $\sim 2$ to avoid large second-order effects.

\section{DECAY IN THE OPTIMIZED FIELDS CONFIGURATION}

We now choose the values of $\alpha_{1}, \alpha_{2}$ marked in Fig. 3(c) to study more precisely the deexcitation mechanism. Before doing so we stress that the choice of the initial states created by the addition of the electric field is not obvious. It is beyond the scope of this paper to study in detail the dynamical behavior of the states mixing during the application of the electric field. We can nevertheless mention that, in order to fully mix the levels, it should not be switched on too fast (meaning in a fully diabatic manner). In the case of our real and nonoscillating Hamiltonian, the adiabaticity criterion to stay in the eigenstates $|n\rangle=\left|n m_{1} m_{2}\right\rangle_{\omega_{1}, \omega_{2}}$ is the standard criterion (with simplified obvious notations) $\sum_{m \neq n} \frac{\hbar\left|\left\langle m\left|d V_{1} / d t\right| n\right\rangle\right|}{\left|E_{n}-E_{m}\right|^{2}}$ [24]. It can be estimated using simple classical vector arguments [25]: the rotation rate of $\omega_{i}$ must always be slow compared to the precession rate $\boldsymbol{\omega}_{i} \cdot \boldsymbol{I}_{i} / \hbar$ (for $i=1,2$ ). Such estimation leads to a rate in the high range of $\mathrm{V} / \mathrm{cm}$ per ns. Thus a rising time less than $\sim 10-100 \mathrm{~ns}$ should be safe to ensure adiabaticity. Because initially antihydrogen is formed in all $\left|n m_{1} m_{2}\right\rangle_{\boldsymbol{B}}$
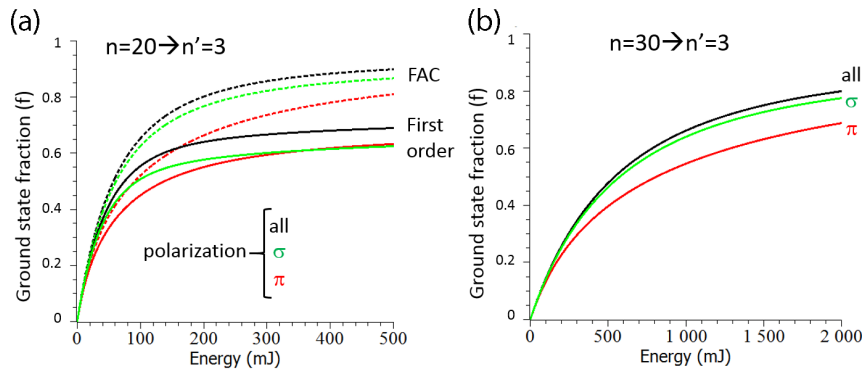

FIG. 4. Fraction $(f)$ of the initial atoms reaching the ground state from the $n=20$ (a) or $n=30$ (b) manifolds after stimulated deexcitation toward $n^{\prime}=3$ in a $1 \mathrm{~T}$ magnetic field and a $280 \mathrm{~V} / \mathrm{cm}$ electric field with $160^{\circ}$ respective angle. Different laser polarizations are considered; see also Fig. 3 (black for isotropic, red for $\pi$, and green for $\sigma^{ \pm}$). The laser has a $5000 \mathrm{GHz}$ linewidth (centered on the $n \rightarrow n^{\prime}=3$ transition) and a waist of $1 \mathrm{~mm}$. In (a) we added the comparison between our calculations using first-order theory (solid lines) and the output of the flexible atomic code (FAC) (dashed lines).

states we will assume adiabaticity and so an equidistribution of the $\left|n m_{1} m_{2}\right\rangle_{\omega_{1}, \omega_{2}}$ states before the application of laser deexcitation.

As before, we assume an abrupt application of a laser of duration $t=100 \mathrm{~ns}$. The total population transfer toward the ground state is plotted in Fig. 4 as a function of the laser power for two initial $n$ manifold ( $n=20$ and $n=30$ ) and three laser polarizations: $\sigma^{ \pm}, \pi$, or isotropic. More precisely the total population transfer is calculated as the sum of the population transfer of the initially populated $\left|n m_{1} m_{2}\right\rangle$ levels: $\frac{1}{n^{2}} \sum_{m_{1}, m_{2}} P_{n m_{1} m_{2}}^{(q)}(t) . P_{n m_{1} m_{2}}^{(q)}(t)$ is calculated using Eqs. (5) with the stimulated decay rate $\Gamma_{\text {stim }}^{n m_{1} m_{2} ; n^{\prime} ; q}$, the photoionization rate of the $n m_{1} m_{2}$ levels to the states of energy $k$ in the continuum (determined by the laser central wavelength) $\Gamma_{\text {photo }}$ and the spontaneous emission rate $\Gamma_{\text {spon }}=1 / \tau$.

For $n=30$ to $n^{\prime}=3$, an efficient transfer of more $80 \%$ is obtained for a $2 \mathrm{~J}$ laser energy. For the $n=20$ case the transfer requires, as expected, less laser energy because of the larger transition dipole moment. But despite the fact that the field configuration is not optimal for $n=20$ (in that case we have the following values: $\alpha_{1}=0.151$ and $\alpha_{2}=0.756$ ), we still obtain around $60 \%$ transfer. In order to study the validity of our assumptions we perform the exact diagonalization of the Hamiltonian using the flexible atomic code (FAC: a software package for the calculation of various atomic processes [26]) for the same field configuration and including all states in the $n=1,3,20$ manifolds as well as the photoionization to the continuum. The code takes into account the fine structure and the full Stark and Zeeman effects (including the quadratic Zeeman effect). We compare in Fig. 4(a) those results to our first-order calculation. A relatively good agreement is found. Interestingly, FAC predicts an even better mixing (which might be due to the quadratic Zeeman effect) showing that our estimated deexcitation efficiencies are probably slightly underestimated. 


\section{CONCLUSION}

We find that adding an electric field to the magnetic field already present in the antihydrogen apparatus will allow us to very efficiently stimulate the decay of Rydberg states toward the ground state. A continuous deexcitation might be possible using an intense $\mathrm{CO}_{2}$ laser (that could also be used to directly create the antihydrogen atoms through stimulated radiative recombination [27-29]). A pulsed deexcitation toward $n^{\prime}=3$ can be achieved using a heated alexandrite laser or toward $n^{\prime}=4$ using an amplified OPG laser. For narrower $n$ distribution, the laser power requirement will be more favorable because a smaller bandwidth will be required and thus less laser power would be needed to drive the transitions; the photoionization would also be reduced in the same ratio. In order to further reduce the laser power we could also consider modifying the electric field strength during the laser pulse in order to more efficiently mix all levels. It may also be possible to mix the states using a radio-frequency field resonant to $m \rightarrow m \pm 1$ magnetic transitions or to add $\mathrm{THz}$ sources to stimulate the transitions $n \rightarrow n-1$ between Rydberg states [12].

Once in the ground state the atoms can be manipulated and reexcited to a well-defined state for targeted manipulations. The presented method will thus be very useful in mechanisms which envision the creation of an intense antihydrogen beam via well-controlled Stark acceleration [30] or magnetic focusing [31]. It can also allow a better trapping efficiency if a subsequent excitation is done in a state with a high magnetic moment (this will be the subject of an upcoming publication [12]). We therefore think that our proposal opens exciting future prospects to enhance the production of useful antihydrogen atoms.

\section{APPENDIX}

We find it useful to recall here some basic formulas to calculate hydrogen properties especially because overlap of the radial wave functions of hydrogen, if well known [32], have often been misprinted in several articles (Eqs. (25)-(27) of [33], (2.34) of [34], and (27) of [32]) and textbooks (Eq. (3.17) of [35]).

\section{Radial wave function}

We use calligraphic notation $\left(\mathcal{R}_{n l}\right.$ or $\left.\mathcal{R}_{E l}\right)$ for SI units and the usual typography $\left(R_{n l}\right.$ or $\left.R_{E l}\right)$ for atomic units.

The wave function of hydrogen atoms for bound states of energy $E=-\frac{R y}{n^{2}}$, where $R y=h c R_{\infty}\left(R_{\infty}=\frac{m_{e} c \alpha^{2}}{2 h}\right)$ is the
Rydberg energy, is given by $\psi_{n l m}(r, \theta, \phi)=\mathcal{R}_{n l}(r) Y_{l m}(\theta, \phi)$ with $\mathcal{R}_{n l}(r)=\frac{1}{a_{0}^{3 / 2}} R_{n l}\left(r / a_{0}\right), \rho=r / a_{0}$, and

$$
\begin{aligned}
R_{n l}(\rho)= & \frac{2}{n^{2}} e^{-\frac{\rho}{n}} \sqrt{\frac{(n-l-1) !}{(l+n) !}\left(\frac{2 \rho}{n}\right)^{l} L_{n-l-1}^{2 l+1}\left(\frac{2 \rho}{n}\right)} \\
= & \frac{2}{n^{2}(2 l+1) !} e^{-\frac{\rho}{n}} \sqrt{\frac{(l+n) !}{(n-l-1) !}}\left(\frac{2 \rho}{n}\right)^{l} \\
& \times{ }_{1} F_{1}\left(l-n+1 ; 2 l+2 ; \frac{2 \rho}{n}\right) .
\end{aligned}
$$

The functions are normalized $1=\int_{0}^{\infty} R_{n l}(r)^{2} r^{2} d r=$ $\int_{0}^{\infty} \mathcal{R}_{n l}(\rho)^{2} \rho^{2} d \rho$.

For continuum states $\psi_{E l m}(r, \theta, \phi)=\mathcal{R}_{E l}(r) Y_{l m}(\theta, \phi)$ with energy $E=k^{2} R y$.

Several normalizations (in energy, wave number, $k^{2}, \ldots$ ) are possible [36]. We choose here the energy normalization $\delta\left(E-E^{\prime}\right)=\int_{0}^{\infty} \mathcal{R}_{E l}(r) r^{2} \mathcal{R}_{E^{\prime} l}(r) d r$. So with $\mathcal{R}_{E l}(r)=$ $\frac{1}{R y^{1 / 2} a_{0}^{3 / 2}} R_{k l}\left(r / a_{0}\right)$ we have the normalization through $\delta\left(k^{2}-\right.$ $\left.k^{\prime 2}\right)=\int_{0}^{\infty} R_{k l}(\rho) \rho^{2} R_{k^{\prime} l}(\rho) d r$ (so with a factor $\pi$ different compared to Ref. [33]). We have (up to a phase factor)

$$
\begin{aligned}
R_{k l}(\rho)= & \frac{1}{(2 l+1) !} e^{i k \rho} \sqrt{\frac{2 \prod_{s=0}^{l}\left(1+s^{2} k^{2}\right)}{1-e^{-\frac{2 \pi}{k}}}}(2 \rho)^{l} \\
& \times{ }_{1} F_{1}\left(l-\frac{i}{k}+1 ; 2 l+2 ;-2 i k \rho\right) .
\end{aligned}
$$

The wave functions are similar to the bound state ones (through the modification $n \rightarrow i k$ due to the energy definition) $[36,37]$.

\section{Reduced dipole matrix element}

The Wigner-Eckart theorem indicates that (bound-bound or bound-continuum) dipole $\boldsymbol{d}=e \boldsymbol{r}$ matrix elements between $|n l m\rangle$ and $\left|n^{\prime} l^{\prime} m^{\prime}\right\rangle$ states (or with $k$ in place of $n^{\prime}$ for continuum states) are given by

$$
\begin{aligned}
\left\langle n^{\prime} l^{\prime} m^{\prime}\left|r^{(q)} / a_{0}\right| n l m\right\rangle & =C_{l m, 1 q}^{l^{\prime} m^{\prime}} \frac{\left\langle n^{\prime} l^{\prime}\left\|r / a_{0}\right\| n l\right\rangle}{\sqrt{2 l^{\prime}+1}} \\
& =C_{l m, 1 q}^{l^{\prime} m^{\prime}} C_{l 0,10}^{l^{\prime} 0} \frac{\sqrt{2 l+1}}{\sqrt{2 l^{\prime}+1}} R_{n l}^{n^{\prime} l^{\prime}} .
\end{aligned}
$$

The overlap $R_{n l}^{n^{\prime} l^{\prime}}=\int_{0}^{\infty} R_{n l}(\rho) \rho R_{n^{\prime} l^{\prime}}(\rho) \rho^{2} d \rho=\mathcal{R}_{n l}^{n^{\prime} l^{\prime}} / a_{0}$ is directly the atomic unit value:

$$
\begin{aligned}
R_{n l}^{n^{\prime} l-1}= & \frac{(-1)^{n^{\prime}-l}}{4(2 l-1) !} \sqrt{\frac{(l+n) !\left(l+n^{\prime}-1\right) !}{(-l+n-1) !\left(n^{\prime}-l\right) !} \frac{\left(4 n n^{\prime}\right)^{l+1}\left(n-n^{\prime}\right)^{n+n^{\prime}-2 l-2}}{\left(n+n^{\prime}\right)^{n+n^{\prime}}}} \\
& \times\left[{ }_{2} F_{1}\left(l-n+1, l-n^{\prime}, 2 l,-\frac{4 n n^{\prime}}{\left(n-n^{\prime}\right)^{2}}\right)-\frac{\left(n-n^{\prime}\right)^{2}}{\left(n^{\prime}+n\right)^{2}}{ }_{2} F_{1}\left(l-n-1, l-n^{\prime}, 2 l,-\frac{4 n n^{\prime}}{\left(n-n^{\prime}\right)^{2}}\right)\right] .
\end{aligned}
$$

One common case is when the atoms are evenly distributed among all $2 l+1$ possible $m$ initial states. Using the sum rule $\sum_{m q}\left|\left\langle n^{\prime} l^{\prime} m^{\prime}\left|r^{(q)}\right| n l m\right\rangle\right|^{2}=\frac{\left|\left\langle n^{\prime} l^{\prime}\|r\| n l\right\rangle\right|^{2}}{2 l^{\prime}+1}$ we see that for unpolarized light (intensity $1 / 3$ for $\sigma^{+}, 1 / 3$ for $\pi, 1 / 3$ for 
$\sigma^{-}$transition) the probability transition is independent of the initial state. Another useful sum rule is $\sum_{m^{\prime} q}\left|\left\langle n^{\prime} l^{\prime} m^{\prime}\left|r^{(q)}\right| n l m\right\rangle\right|^{2}=\frac{\left|\left\langle n^{\prime} l^{\prime}\|r\| n l\right\rangle\right|^{2}}{2 l+1}$.

\section{Spontaneous system}

The spontaneous emission rate between an $n l m$ and $n^{\prime} l^{\prime} m^{\prime}$ level with photon angular frequency $\omega$ is given by

$$
A_{n l m}^{n^{\prime} l^{\prime} m}=\frac{\omega^{3} e^{2} a_{0}^{2}}{3 \pi \varepsilon_{0} \hbar c^{3}}\left|\left\langle n^{\prime} l^{\prime} m^{\prime}\left|r^{\left(q=m^{\prime}-m\right)} / a_{0}\right| n l m\right\rangle\right|^{2} .
$$

So when summed over the final states the spontaneous emission rate between an $n l m$ level and (all) $n^{\prime} l^{\prime}$ levels is given by $A_{n l m}^{n^{\prime} l^{\prime}}=\frac{e^{2} a_{0}^{2} \omega^{3}}{3 \pi \varepsilon_{0} \hbar c^{3}} \frac{\left|\left\langle n^{\prime} l^{\prime}\left\|r / a_{0}\right\| n l\right\rangle\right|^{2}}{2 l+1}$ which does not depend on $m$ and can be noted $A_{n l}^{n^{\prime} l^{\prime}}$ the spontaneous emission rate from an $n l$ toward all $n^{\prime} l^{\prime}$ levels. Using $\hbar \omega=\frac{R y}{n^{\prime 2}}-\frac{R y}{n^{2}}$ we find

$$
\begin{aligned}
A_{n l}^{n^{\prime} l^{\prime}} & =\frac{e^{2} \omega^{3}}{3 \pi \varepsilon_{0} \hbar c^{3}} \frac{\max \left(l, l^{\prime}\right)}{2 l+1}\left(\mathcal{R}_{n^{\prime} l^{\prime}}^{n l}\right)^{2} \\
& =\frac{\alpha^{4} c}{6 a_{0}}\left(\frac{1}{n^{\prime 2}}-\frac{1}{n^{2}}\right)^{3} \frac{\max \left(l, l^{\prime}\right)}{2 l+1}\left(R_{n l}^{n^{\prime} l^{\prime}}\right)^{2} \\
& =\frac{e^{2} a_{0}^{2} \omega^{3}}{3 \pi \varepsilon_{0} \hbar c^{3}} \frac{\left|\left\langle n^{\prime} l^{\prime}\left\|r / a_{0}\right\| n l\right\rangle\right|^{2}}{2 l+1} \\
& =\frac{A_{n l m}^{n^{\prime} l^{\prime} m}}{\left(C_{l m, 1 q=m^{\prime}-m}^{l^{\prime} m^{\prime}}\right)^{2} \frac{2 l+1}{2 l^{\prime}+1}}=\frac{A_{n l m}^{n^{\prime} l^{\prime} m^{\prime}}}{\left(C_{l^{\prime} m^{\prime}, 1(-q)=\left(m-m^{\prime}\right)}^{l m}\right)^{2}} .
\end{aligned}
$$

\section{Stimulated emission rate $\Gamma^{\prime}$}

The stimulated emission rate $\Gamma^{\prime}$ between an excited $\mathrm{nlm}$ and $n^{\prime} l^{\prime} m^{\prime}$ level can be calculated in the same way. The general formula for 2 levels $e$ and $g$ (separated in energy by $\hbar \omega_{\mathrm{eg}}$, a dipole $d_{\text {eg }}=e a_{0}\left\langle n^{\prime} l^{\prime} m^{\prime}\left|r^{\left(q=m-m^{\prime}\right)} / a_{0}\right| n l m\right\rangle$ transition, and a laser polarization vector $\boldsymbol{\epsilon})$ is

$$
\Gamma^{\prime}=\frac{\int L(\omega) I(\omega) d \omega \pi\left|\boldsymbol{d}_{\mathrm{eg}} \cdot \boldsymbol{\epsilon}\right|^{2}}{\hbar^{2} \epsilon_{0} c}
$$

where $L(\omega)=\frac{1}{\pi} \frac{\Gamma / 2}{\left(\omega-\omega_{\mathrm{eg}}\right)^{2}+(\Gamma / 2)^{2}}$ is the Lorentzian spectral spectrum for the spontaneous emission and $I(\omega)$ is the laser irradiance spectral distribution (throughout the article we use improperly the word intensity).

For example for a Lorentzian spectrum $I(\omega)=$ $\frac{I}{\pi} \frac{\Gamma_{\mathrm{L}} / 2}{\left(\omega-\omega_{0}\right)^{2}+\left(\Gamma_{\mathrm{L}} / 2\right)^{2}}\left[I=\int I(\omega) d \omega\right.$ is the full laser irradiance so the laser electric field is $E=\sqrt{2 I / \epsilon_{0} c}$, we have $\Gamma^{\prime}=\frac{\Omega^{2} / 2}{\left(\omega_{0}-\omega_{\mathrm{eg}}\right)^{2}+\left[\left(\Gamma_{\mathrm{L}}+\Gamma\right) / 2\right]^{2}} \frac{\Gamma_{\mathrm{L}}+\Gamma}{2}$, where $\Omega=\mathbf{d}_{\mathrm{eg}} \cdot \mathbf{E} / \hbar$ is the Rabi frequency.

For a broadband laser, where $\Gamma_{\mathrm{L}} \gg \Gamma$, the final rate at resonance is

$$
\Gamma^{\prime}=\frac{\Omega^{2}}{\Gamma_{\mathrm{L}}}=\frac{2 I\left|d_{\mathrm{eg}} \epsilon_{q}\right|^{2}}{\hbar^{2} \epsilon_{0} c \Gamma_{\mathrm{L}}} .
$$

Therefore an average rate for a fully mixed state $\left|\psi_{n}\right\rangle \approx \sum_{l m} 1 / n|n l m\rangle$ under an isotropic (unpolarized) light is given by the sum over all $l^{\prime} m^{\prime}$ transition rates and so is $\Gamma^{\prime}=\frac{2 I e^{2} a_{0}^{2}}{\hbar^{2} \epsilon_{0} c \Gamma_{\mathrm{L}}} \frac{1}{3} \sum_{q} \sum_{l^{\prime} m^{\prime}}\left|\left\langle\psi_{n}\left|r^{(q)} / a_{0}\right| n^{\prime} l^{\prime} m^{\prime}\right\rangle\right|^{2}=$ $\frac{2 I e^{2} a_{0}^{2}}{\hbar^{2} \epsilon_{0} c \Gamma_{\mathrm{L}}} \frac{1}{3 n^{2}} \sum_{l m} \sum_{q} \sum_{l^{\prime} m^{\prime}}\left|\left\langle n l m\left|r^{(q)} / a_{0}\right| n^{\prime} l^{\prime} m^{\prime}\right\rangle\right|^{2}=$ $\frac{2 I e^{2} a_{0}^{2}}{\hbar^{2} \epsilon_{0} c \Gamma_{\mathrm{L}}} \frac{1}{3 n^{2}} \sum_{l^{\prime} l}\left|\left\langle n^{\prime} l^{\prime}\left\|r / a_{0}\right\| n l\right\rangle\right|^{2}$.

\section{Photoionization cross section}

Using $R_{n l}^{k l^{\prime}}=\int_{0}^{\infty} R_{n l}(\rho) \rho R_{k l^{\prime}}(\rho) \rho^{2} d \rho$, the photoionization cross section from $n l$ to $k l^{\prime}$ is given by

$$
\begin{aligned}
\sigma_{n l}^{k l^{\prime}} & =\frac{4 \pi^{2} \omega a_{0} 2 R y}{3 c} \frac{\max \left(l, l^{\prime}\right)}{2 l+1}\left(\mathcal{R}_{n l}^{k l^{\prime}}\right)^{2} \\
& =\frac{4 \pi^{2} \alpha a_{0}^{2}}{3}\left(\frac{1}{n^{2}}+k^{2}\right) \frac{\max \left(l, l^{\prime}\right)}{2 l+1}\left(R_{n l}^{k l^{\prime}}\right)^{2} \\
& =\frac{4 \pi^{2} \alpha a_{0}^{2}}{3}\left(\frac{1}{n^{2}}+k^{2}\right) \frac{\left|\left\langle k l^{\prime}\left\|r / a_{0}\right\| n l\right\rangle\right|^{2}}{2 l+1} .
\end{aligned}
$$

It is the cross section assuming that the atoms are evenly distributed among all $2 l+1$ possible $m$ initial states. So, for a light of given polarization $q, \sigma_{n l}^{k l^{\prime}}=\frac{1}{2 l+1} \sum_{m} \sigma_{n l m}^{k l^{\prime} m^{\prime}=m+q}$, where $\sigma_{n l m}^{k l^{\prime} m^{\prime}=m+q}$ is the photoionization cross section from $n l m$ to $k l^{\prime} m^{\prime}$ given by

$$
\begin{aligned}
\sigma_{n l m}^{k l^{\prime} m^{\prime}} & =3\left(C_{l m, 1 q=m^{\prime}-m}^{l^{\prime} m^{\prime}}\right)^{2} \frac{2 l+1}{2 l^{\prime}+1} \sigma_{n l}^{k l^{\prime}} \\
& =3\left(C_{l^{\prime} m^{\prime}, 1 m-m^{\prime}}^{l m}\right)^{2} \sigma_{n l}^{k l^{\prime}} \\
& =4 \pi^{2} \alpha a_{0}^{2}\left(\frac{1}{n^{2}}+k^{2}\right)\left|\left\langle k l^{\prime} m^{\prime}\left|r^{\left(m^{\prime}-m\right)} / a_{0}\right| n l m\right\rangle\right|^{2} .
\end{aligned}
$$

$R_{n l}^{k l^{\prime}}$ is given for $l^{\prime}=l+1$ and for $l^{\prime}=l-1$ by

$$
\begin{aligned}
R_{n l}^{k l+1}= & \frac{-i}{4 k(2 l+1) !} \sqrt{\frac{1}{2} \frac{(n+l) ! \prod_{s=1}^{l+1}\left(1+s^{2} k^{2}\right)}{(n-l-1) !\left(1-e^{-\frac{2 \pi}{k}}\right)}}\left(\frac{4 n}{1+n^{2} k^{2}}\right)^{l+2} e^{-\frac{2}{k} \arctan (n k)}\left(\frac{n-i / k}{n+i / k}\right)^{n-l-2} \\
& \times\left[{ }_{2} F_{1}\left(l+2-i / k, l+1-n ; 2 l+2 ;-\frac{4 n i / k}{(n-i / k)^{2}}\right)\right. \\
& \left.-\left(\frac{n-i / k}{n+i / k}\right)_{2}^{2} F_{1}\left(l-i / k, l+1-n ; 2 l+2 ;-\frac{4 n i / k}{(n-i / k)^{2}}\right)\right],
\end{aligned}
$$




$$
\begin{aligned}
R_{n l}^{k l-1}= & \frac{-1}{4(2 l+1) !} \sqrt{\frac{1}{2} \frac{(n+l) ! \prod_{s=1}^{l-1}\left(1+s^{2} k^{2}\right)}{(n-l-1) !\left(1-e^{-\frac{2 \pi}{k}}\right)}}\left(\frac{4 n}{1+n^{2} k^{2}}\right)^{l+1} e^{-\frac{2}{k} \arctan (n k)}\left(\frac{n-i / k}{n+i / k}\right)^{n-l-1} \\
& \times\left[{ }_{2} F_{1}\left(l-i / k, l+1-n ; 2 l ;-\frac{4 n i / k}{(n-i / k)^{2}}\right)-\left(\frac{n-i / k}{n+i / k}\right)^{2}{ }_{2} F_{1}\left(l-i / k, l-1-n ; 2 l ;-\frac{4 n i / k}{(n-i / k)^{2}}\right)\right] .
\end{aligned}
$$

We do not treat here the continuum-continuum transition (it is given in [32]).

[1] C. J. Baker, W. Bertsche, A. Capra, C. Carruth, C. L. Cesar, M. Charlton, S. Cohen, R. Collister, S. Eriksson, A. Evans, N. Evetts, J. Fajans, T. Friesen, M. C. Fujiwara, D. R. Gill, J. S. Hangst, W. N. Hardy, M. E. Hayden, C. A. Isaac, M. A. Johnson, J. M. Jones, S. A. Jones, S. Jonsell, A. Khramov, P. Knapp, L. Kurchaninov, N. Madsen, D. Maxwell, J. T. K. McKenna, S. Menary, T. Momose, J. J. Munich, K. Olchanski, A. Olin, P. Pusa, C. Rasmussen, F. Robicheaux, R. L. Sacramento, M. Sameed, E. Sarid, D. M. Silveira, G. Stutter, C. So, T. D. Tharp, R. I. Thompson, D. P. van der Werf, J. S. Wurtele, M. Ahmadi, and B. X. R. Alves, Characterization of the $1 \mathrm{~S}-2 \mathrm{~S}$ transition in antihydrogen, Nature (London) 557, 71 (2018).

[2] M. Ahmadi, M. Baquero-Ruiz, W. Bertsche, E. Butler, A. Capra, C. Carruth, C. L. Cesar, M. Charlton, A. E. Charman, S. Eriksson et al., An improved limit on the charge of antihydrogen from stochastic acceleration, Nature (London) 529, 373 (2016).

[3] M. Ahmadi, B. X. R. Alves, C. J. Baker, W. Bertsche, E. Butler, A. Capra, C. Carruth, C. L. Cesar, M. Charlton, S. Cohen, R. Collister, S. Eriksson, A. Evans, N. Evetts, J. Fajans, T. Friesen, M. C. Fujiwara, D. R. Gill, A. Gutierrez, J. S. Hangst, W. N. Hardy, M. E. Hayden, C. A. Isaac, A. Ishida, M. A. Johnson, S. A. Jones, S. Jonsell, L. Kurchaninov, N. Madsen, M. Mathers, D. Maxwell, J. T. K. McKenna, S. Menary, J. M. Michan, T. Momose, J. J. Munich, P. Nolan, K. Olchanski, A. Olin, P. Pusa, C. Ø. Rasmussen, F. Robicheaux, R. L. Sacramento, M. Sameed, E. Sarid, D. M. Silveira, S. Stracka, G. Stutter, C. So, T. D. Tharp, J. E. Thompson, R. I. Thompson, D. P. van der Werf, and J. S. Wurtele, Observation of the hyperfine spectrum of antihydrogen, Nature (London) 548, 66 (2017).

[4] F. Robicheaux, Atomic processes in antihydrogen experiments: A theoretical and computational perspective, J. Phys. B 41, 192001 (2008).

[5] B. Radics, D. J. Murtagh, Y. Yamazaki, and F. Robicheaux, Scaling behavior of the ground-state antihydrogen yield as a function of positron density and temperature from classicaltrajectory Monte Carlo simulations, Phys. Rev. A 90, 032704 (2014).

[6] M. Ahmadi, B. X. R. Alves, C. J. Baker, W. Bertsche, E. Butler, A. Capra, C. Carruth, C. L. Cesar, M. Charlton, S. Cohen, R. Collister, S. Eriksson, A. Evans, N. Evetts, J. Fajans, T. Friesen, M. C. Fujiwara, D. R. Gill, A. Gutierrez, J. S. Hangst, W. N. Hardy, M. E. Hayden, C. A. Isaac, A. Ishida, M. A. Johnson, S. A. Jones, S. Jonsell, L. Kurchaninov, N. Madsen, M. Mathers, D. Maxwell, J. T. K. McKenna, S. Menary, J. M. Michan, T. Momose, J. J. Munich, P. Nolan, K. Olchanski, A. Olin, P. Pusa, C. Ø. Rasmussen, F. Robicheaux, R. L. Sacramento,
M. Sameed, E. Sarid, D. M. Silveira, S. Stracka, G. Stutter, C. So, T. D. Tharp, J. E. Thompson, R. I. Thompson, D. P. van der Werf, and J. S. Wurtele, Antihydrogen accumulation for fundamental symmetry tests, Nat. Commun. 8, 681 (2017).

[7] C. Malbrunot, C. Amsler, S. Arguedas Cuendis, H. Breuker, P. Dupre, M. Fleck, H. Higaki, Y. Kanai, B. Kolbinger, N. Kuroda, M. Leali, V. Mckel, V. Mascagna, O. Massiczek, Y. Matsuda, Y. Nagata, M. C. Simon, H. Spitzer, M. Tajima, S. Ulmer, L. Venturelli, E. Widmann, M. Wiesinger, Y. Yamazaki, and J. Zmeskal, The ASACUSA antihydrogen and hydrogen program: Results and prospects, Philos. Trans. R. Soc. London A 376, 20170273 (2018).

[8] M. R. Flannery and D. Vrinceanu, Quantal and classical radiative cascade in Rydberg plasmas, Phys. Rev. A 68, 030502 (2003).

[9] T. Topçu and F. Robicheaux, Radiative cascade of highly excited hydrogen atoms in strong magnetic fields, Phys. Rev. A 73, 043405 (2006).

[10] M. A. Henry and F. Robicheaux, Simulation of motion and radiative decay of Rydberg hydrogen atoms in electric and magnetic fields, J. Phys. B 44, 145003 (2011).

[11] D. Krasnický, R. Caravita, C. Canali, and G. Testera, Cross section for Rydberg antihydrogen production via charge exchange between Rydberg positroniums and antiprotons in a magnetic field, Phys. Rev. A 94, 022714 (2016).

[12] M. Vieille-Grosjean, E. Dimova, Z. Mazzotta, D. Comparat, T. Wolz, and C. Malbrunot, Stimulated decay of antihydrogen atoms using selective $\mathrm{THz}$ light and cooling in a magnetic trap (unpublished).

[13] S. Aghion, C. Amsler, G. Bonomi, R. S. Brusa, M. Caccia, R. Caravita, F. Castelli, G. Cerchiari, D. Comparat, G. Consolati, A. Demetrio, L. Di Noto, M. Doser, C. Evans, M. Fanì, R. Ferragut, J. Fesel, A. Fontana, S. Gerber, M. Giammarchi, A. Gligorova, F. Guatieri, S. Haider, A. Hinterberger, H. Holmestad, A. Kellerbauer, O. Khalidova, D. Krasnický, V. Lagomarsino, P. Lansonneur, P. Lebrun, C. Malbrunot, S. Mariazzi, J. Marton, V. Matveev, Z. Mazzotta, S. R. Müller, G. Nebbia, P. Nedelec, M. Oberthaler, N. Pacifico, D. Pagano, L. Penasa, V. Petracek, F. Prelz, M. Prevedelli, B. Rienaecker, J. Robert, O. M. Røhne, A. Rotondi, H. Sandaker, R. Santoro, L. Smestad, F. Sorrentino, G. Testera, I. C. Tietje, E. Widmann, P. Yzombard, C. Zimmer, J. Zmeskal, N. Zurlo, and M. Antonello, Compression of a mixed antiproton and electron non-neutral plasma to high densities, Eur. Phys. J. D 72, 76 (2018).

[14] C.-Y. Wan, U. Werling, and K. F. Renk, Study of broadband emission of an ultraviolet preionized 20-atm $\mathrm{CO}_{2}$ laser, J. Appl. Phys. 57, 990 (1985). 
[15] P. K. Gupta and U. K. Chatterjee, Various techniques for multiline operation of TEA $\mathrm{CO}_{2}$ lasers, Opt. Laser Technol. 22, 403 (1990)

[16] S. Aghion, C. Amsler, A. Ariga, T. Ariga, G. Bonomi, P. Bräunig, J. Bremer, R. S. Brusa, L. Cabaret, M. Caccia, R. Caravita, F. Castelli, G. Cerchiari, K. Chlouba, S. Cialdi, D. Comparat, G. Consolati, A. Demetrio, L. Di Noto, M. Doser, A. Dudarev, A. Ereditato, C. Evans, R. Ferragut, J. Fesel, A. Fontana, O. K. Forslund, S. Gerber, M. Giammarchi, A. Gligorova, S. Gninenko, F. Guatieri, S. Haider, H. Holmestad, T. Huse, I. L. Jernelv, E. Jordan, A. Kellerbauer, M. Kimura, T. Koettig, D. Krasnicky, V. Lagomarsino, P. Lansonneur, P. Lebrun, S. Lehner, J. Liberadzka, C. Malbrunot, S. Mariazzi, L. Marx, V. Matveev, Z. Mazzotta, G. Nebbia, P. Nedelec, M. Oberthaler, N. Pacifico, D. Pagano, L. Penasa, V. Petracek, C. Pistillo, F. Prelz, M. Prevedelli, L. Ravelli, L. Resch, B. Rienäcker, O. M. Røhne, A. Rotondi, M. Sacerdoti, H. Sandaker, R. Santoro, P. Scampoli, L. Smestad, F. Sorrentino, M. Spacek, J. Storey, I. M. Strojek, G. Testera, I. Tietje, S. Vamosi, E. Widmann, P. Yzombard, J. Zmeskal, and N. Zurlo, Laser excitation of the $n=3$ level of positronium for antihydrogen production, Phys. Rev. A 94, 012507 (2016).

[17] H. Samelson, D. Harter, J. Pete, R. Morris, J. Walling, and D. Heller, Tunable alexandrite lasers: Development and performance, IEEE J. Quantum Electron. 21, 1568 (1985).

[18] J. W. Kuper, T. Chin, and H. E. Aschoff, Extended tuning range of alexandrite at elevated temperatures, in Advanced Solid State Lasers (Optical Society of America, Washington, DC, 1990), p. CL3.

[19] E. A. Solovev, Second order perturbation theory for the hydrogen atom in crossed electric and magnetic fields, Zh. Eksp. Teor. Fiz. 85, 109 (1983).

[20] J. Main, M. Schwacke, and G. Wunner, Hydrogen atom in combined electric and magnetic fields with arbitrary mutual orientations, Phys. Rev. A 57, 1149 (1998).

[21] P. A. Braun, Discrete semiclassical methods in the theory of Rydberg atoms in external fields, Rev. Mod. Phys. 65, 115 (1993).

[22] Y. N. Demkov, B. S. Monozon, and V. N. Ostrovskii, Energy levels of a hydrogen atom in crossed electric and magnetic fields, Sov. Phys. JETP 30, 775 (1970).
[23] D. A. Varshalovich, A. N. Moskalev, and V. K. Khersonskii, Quantum Theory of Angular Momentum (World Scientific Publishing, Teaneck, NJ, 1987).

[24] D. Comparat, General conditions for quantum adiabatic evolution, Phys. Rev. A 80, 012106 (2009).

[25] R. Lutwak, J. Holley, P. P. Chang, S. Paine, D. Kleppner, and T. Ducas, Circular states of atomic hydrogen, Phys. Rev. A 56, 1443 (1997).

[26] M. F. Gu, The flexible atomic code, Can. J. Phys. 86, 675 (2008).

[27] A. Wolf, Laser-stimulated formation and stabilization of antihydrogen atoms, Hyperfine Interact. 76, 189 (1993).

[28] A. Müller and A. Wolf, Production of antihydrogen by recombination of $\bar{p}$ with $\mathrm{e}^{+}$: What can we learn from electron-ion collision studies? Hyperfine Interact. 109, 233 (1997).

[29] M. Amoretti, C. Amsler, G. Bonomi, P. D. Bowe, C. Canali, C. Carraro, C. L. Cesar, M. Charlton, A. M. Ejsing, A. Fontana et al., Search for Laser-Induced Formation of Antihydrogen Atoms, Phys. Rev. Lett. 97, 213401 (2006).

[30] AEgIS Collaboration, Proposal for the AEgIS experiment at the CERN antiproton decelerator, CERN/SPSC 2007-017, SPSCP-334, 2007.

[31] ASACUSA Collaboration, ASACUSA proposal addendum, CERN/SPSC 2005-002, SPSC P-307, Addendum 1, 2005.

[32] W. Gordon, Zur berechnung der matrizen beim wasserstoffatom, Ann. Phys. 394, 1031 (1929).

[33] A. Burgess, Tables of hydrogenic photoionization crosssections and recombination coefficients, Mem. R. Astron. Soc. 69, 1 (1965).

[34] P. J. Storey and D. G. Hummer, Fast computer evaluation of radiative properties of hydrogenic systems, Comput. Phys. Commun. 66, 129 (1991).

[35] H. A. Bethe and E. E. Salpeter, in Quantum Mechanics of Oneand Two-Electron Atoms (Springer, Boston, MA, 2012).

[36] L. D. Landau and E. M. Lifshitz, Course of Theoretical Physics, Vol. 3, Quantum Mechanics (Pergamon Press, Oxford, 1977).

[37] B. Blaive and M. Cadilhac, A comparison of the hydrogenlike dipole radial matrix elements with overlap integrals and a step toward explicit expressions of the multipole matrix elements, J. Phys. B 42, 165002 (2009). 